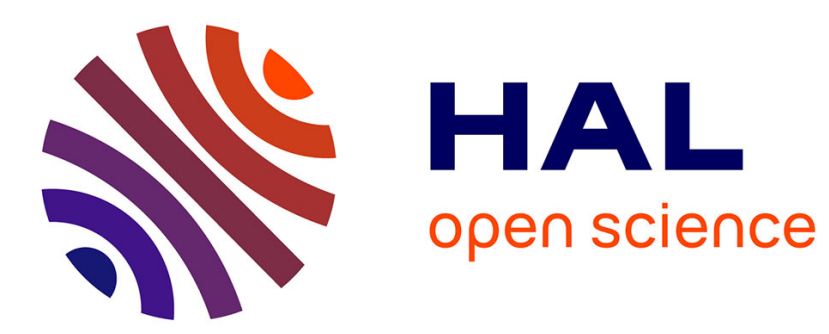

\title{
Average Number of Significant Modes Excited in a Mode-Stirred Reverberation Chamber
}

\author{
Florian Monsef, Andréa Cozza
}

\section{To cite this version:}

Florian Monsef, Andréa Cozza. Average Number of Significant Modes Excited in a Mode-Stirred Reverberation Chamber. IEEE Transactions on Electromagnetic Compatibility, 2014, 56 (2), pp.259265. 10.1109/TEMC.2013.2284924 . hal-00933765

\section{HAL Id: hal-00933765 \\ https://hal-centralesupelec.archives-ouvertes.fr/hal-00933765}

Submitted on 21 Jan 2014

HAL is a multi-disciplinary open access archive for the deposit and dissemination of scientific research documents, whether they are published or not. The documents may come from teaching and research institutions in France or abroad, or from public or private research centers.
L'archive ouverte pluridisciplinaire HAL, est destinée au dépôt et à la diffusion de documents scientifiques de niveau recherche, publiés ou non, émanant des établissements d'enseignement et de recherche français ou étrangers, des laboratoires publics ou privés. 


\title{
Average number of significant modes excited in a mode-stirred reverberation chamber
}

\author{
Florian Monsef, Member IEEE, Andrea Cozza, Senior Member IEEE
}

\begin{abstract}
Although the number of significant modes is intuitive, this concept has never been clearly defined, and this, mainly because of the unbound number of modes involved in modal overlap. In the present paper, we show that, for a perfect stirring process, the effect of modal overlap can be modeled as an equivalent filtering formulation. By introducing the statisticalbandwidth concept we show that the electromagnetic field statistics due to an infinite number of modes can be summarized by a finite number of significant modes. The case of the electric-energy density in an mode-stirred reverberation chamber (MSRC) has been considered and a new expression of its variability has been established. The good agreement found between the new expression and experimental and simulation results support the several concepts introduced in this paper.
\end{abstract}

Index Terms-reverberation chamber (RC), electromagnetic compatibility (EMC), modal analysis, parametric statistics, cavity resonators.

\section{INTRODUCTION}

$\mathbf{M}$ ODE-stirred reverberation chambers (MSRC) are microwave complex environments in which it is of common use to regard the electromagnetic field as a random process. This approach is all the more justified when the chamber is overmoded, i.e., when a large number of modes of the chamber are excited. Indeed, in that case tests indicate that the ratios of peak to average values of the field remain approximately the same [1] [2] [3] throughout the central part of the chamber. However, for metrology purposes the degree of uniformity of such ratios need to be assessed, and this, even for the undermoded case, i.e., near the lowest usable frequency (LUF), for which field statistics differ from the overmoded case.

An important part of the measured-field uncertainty is directly due to the medium complexity. This is why the study of MSRC is based on statistical tools [4] [5] [6] and aims at establishing statistical models able to describe the electromagnetic field from the undermoded to the overmoded regime. The most common approach in the community consists in finding probability density functions $(p d f)$ able to describe the statistics of field-related quantities [7] [8] [9] from the LUF to the overmoded case. An alternative approach consists in modeling the field by using a modal expansion [10] [11]; the $p d f \mathrm{~s}$ to choose are this time those applied to modal parameters. In [10] the derivation was based on a finite number of modes to which a limit to infinity was applied as required by modal theory, whereas in [11] the modal expansion was only including a finite number of contributing modes.

Florian Monsef and Andrea Cozza are with the Départment de Recherche en Electromagnétisme, Laboratoire des Signaux et Systèmes, UMR8506, Univ Paris-Sud, SUPELEC, CNRS, 91190 Gif-sur-Yvette, France.
The notion of contributing mode is intuitively linked to the modal density but the concept has never been clearly defined. In [11] for instance this number was arbitrarily set to the average number of modes overlapping in the average modal bandwidth. In [10], the bandwidth over which this number had to be considered was defined but not quantitatively specified as the aim was to include all the modes. The need to determine this significant number of modes is all the more important that it would provide a metric linked to the degree of overmodedness (or undermodedness) of an MSRC.

To clarify the significant mode concept, we need to regard modal overlap from an alternative point of view; to this end, we will present in section II how the overlap in the frequency domain of an infinite number of modes can be summed up to the frequency response of a single mode. In section III, we will show that the concept of statistical bandwidth provides a statistical criterion that highlights the frequency range over which the number of significant modes must be considered. This finding allows revising the expression of the variability of the electric energy density, referred to as $\varsigma_{W}^{2}$, derived in [10], by highlighting some key terms intervening in its expression. However, the number of significant modes cannot be directly assessed experimentally. This notwithstanding, as shown in section IV, the validation of $\varsigma_{W}^{2}$ 's law allows us to validate the assessment of the number of significant modes.

\section{REVISITING MODAL OVERLAP}

The aim of this section is to revisit the way in which we commonly represent and regard the modal overlap effect. To this end it is convenient to consider the statistics of a fieldrelated quantity, such as the square modulus of the field in an MSRC or, equivalently, its corresponding electric-energy density referred to as $W$. This choice has the advantage to provide a link with a previous work [10] dealing with the problem of considering modal overlap in the derivations of the statistical moments of $W$; to improve the readability with that previous work, we adopt the same notations as much as possible and we invite the reader to refer to the aforementioned paper.

To conduct this study we need to consider a modal approach in which the electric field $\mathbf{E}$ at a position $\mathbf{r}$ and at an operating frequency $f_{w}$ can be expanded as follows,

$$
\mathbf{E}\left(\mathbf{r}, f_{w}\right)=\sum_{n=1}^{\infty} \gamma_{n} \psi_{n}\left(f_{w}\right) \mathbf{e}_{n}(\mathbf{r}),
$$

where $\mathbf{e}_{n}(\mathbf{r})$ is the eigenvector of the $\mathrm{n} t \mathrm{t}$ mode, $\gamma_{n}$ is the modal weight, i.e., the coupling constant of the excitation 
source to the $n$th eigenmode and $\psi_{n}$ is the frequency response of the $n$th mode.

If we express, on the one hand, $\mathbf{e}_{n}(\mathbf{r})=e_{n}(\mathbf{r}) \hat{\xi}_{n}(\mathbf{r})$ where $\hat{\xi}_{n}(\mathbf{r})$ is the unitary polarization vector of the $\mathrm{n} t h$ mode, and use, on the other hand, $\left\{\tilde{\gamma}_{n}\right\}$ referred to as equivalent modal weights and defined as,

$$
\tilde{\gamma}_{n}(\mathbf{r})=\gamma_{n} e_{n}(\mathbf{r})
$$

expression (1) can be expressed in the following convenient form,

$$
\mathbf{E}\left(\mathbf{r}, f_{w}\right)=\sum_{n=1}^{\infty} \tilde{\gamma}_{n}(\mathbf{r}) \psi_{n}\left(f_{w}\right) \hat{\xi}_{n}(\mathbf{r}),
$$

where the frequency response $\psi_{n}(f)$ reads,

$$
\psi_{n}(f)=\frac{-\mathrm{j} f}{f^{2}-f_{n}^{2}-\mathrm{j} f f_{n} / Q_{n}} .
$$

Under the composite quality factor approximation [12], $Q_{n} \simeq$ $Q\left(f_{n}\right)$, where $Q(f)$ is the composite quality factor, a function of frequency. High Q resonances obtained in MSRC are such that the most contributing part of $\psi_{n}(f)$ come from working frequencies "close" to the eigenfrequency $f_{n}$, so that (4) can be approximated by,

$$
\psi_{n}(f) \simeq \frac{2\left(f-f_{n}\right)+\mathrm{j} B_{M}}{4\left(f-f_{n}\right)^{2}+B_{M}^{2}},
$$

where $B_{M}$ is the modal bandwidth related to the composite quality factor. As it will be stressed hereafter $B_{M}$ will be approximated by the modal bandwidth assessed at working frequency, i.e., $B_{M} \simeq f_{w} / Q\left(f_{w}\right)$.

The concept of significant mode is studied here in a statistical sense. To position the problem of modal overlap within this framework, let us consider the average of $W$ (Eq.19 in [10]) expressed herein as follows,

$$
\begin{aligned}
\mathrm{E}[W] & \equiv \mathrm{E}_{f_{n},\left|\tilde{\gamma}_{n}\right|^{2}}[W] \\
& =\mathrm{E}_{f_{n},\left|\tilde{\gamma}_{n}\right|^{2}}\left[\sum_{n=1}^{\infty}\left|\tilde{\gamma}_{n}\right|^{2} \mid \psi_{n}\left(f_{w}\right)\right],
\end{aligned}
$$

where $\mathrm{E}_{x}[\cdot]$ stands for the ensemble-average operator linked to the stirring process and applied to the random variable $x$; note that the spatial dependence of the $\left|\tilde{\gamma}_{n}\right|^{2}$ has been omitted for the sake of brevity.

The difficulty of defining the number of significant modes lies in the infinite number of tails of frequency responses that overlap at working frequency. The upper plot in Fig. 1 depicts the case of a random realization based on a set of frequency responses of excited modes, weighted by a set of equivalent squared modal weights $\left|\tilde{\gamma}_{n}\right|^{2}$ (vertical arrows); the excitation level of the frequency responses at working frequency are highlighted by black dots.

Now, the discrete sum in (6) consists in summing the values indicated by the black dots weighted by the corresponding $\left|\tilde{\gamma}_{n}\right|^{2}$. As already done in [10], each frequency response in Fig. 1 is expressed by means of a frequency template $\psi_{0}(f)$ defined as,

$$
\psi_{n}(f) \simeq \psi_{0}\left(f-f_{n}\right)
$$

The use of such template could appear as a way of simplifying the derivation with a degree of approximation that can be questioned, because of the well-known variation of the composite Q-factor with frequency. In fact, as shown in (4) and (5), the important parameter to consider in $\psi_{n}(f)$ is the modal bandwidth (i.e., the ratio $f_{n} / Q_{n}$ ) which, given the local quasi-linear variation of $Q$ with frequency, does not change significantly. The experimental data presented in section $\mathrm{V}$ show that the deviation of the average modal bandwidth is of $0.7 \%$ over a $100 \mathrm{MHz}$ bandwidth centered about $800 \mathrm{MHz}$. So the results obtained by using (7) are expected to ensure a reasonable level of accuracy.

Using (7) in (6) allows us to approximate the discrete sum (of (6)) as follows,

$$
\sum_{n=1}^{\infty}\left|\tilde{\gamma}_{n}\right|^{2}\left|\psi_{n}\left(f_{w}\right)\right|^{2} \simeq|\tilde{\gamma}(f)|^{2}\left|\psi_{0}\left(f-f_{w}\right)\right|^{2},
$$

where $|\tilde{\gamma}(f)|^{2}$ is a discrete random signal for a given realization.

In order to further recast (6), it is worth recalling that the ensemble average operator stands for an average over an ideally infinite number of stirring states. Moreover, since a stirring process consists in displacing resonance frequencies over a small frequency range, referred to as $\overline{\Delta f}$ (see lower plot of Fig. 1), the vertical dashed line in the upper plot of Fig. 1, as well as the initially discrete frequency template, will both become continuous under perfect stirring conditions.

Note that the random signal $|\tilde{\gamma}(f)|^{2}$ is analog to a power spectrum density (PSD). This one will become continuous in the frequency domain with an average amplitude $\mu_{2}=$ $\mathrm{E}\left[\left|\tilde{\gamma}_{n}\right|^{2}\right]$.

Accordingly the ensemble average over $W$ can be approximated by a frequency average expressed as follows,

$$
\mathrm{E}[W] \simeq \lim _{B_{e} \rightarrow \infty} \frac{1}{\overline{\Delta f}} \int_{\left(B_{e}\right)}|\tilde{\gamma}(f)|^{2}\left|\psi_{0}\left(f-f_{w}\right)\right|^{2} \mathrm{~d} f,
$$

where $B_{e}$ is the frequency range centered about $f_{w}$; an infinite $B_{e}$ allows including all the modes.

If (6) is compared to (9) an ergodic-like property is exhibited. When dealing with the first-order moment, this property consists usually (in its strict sense) in considering that a statistical average coincide with a temporal average; in the present case the statistical average can be approximately assessed by a frequency average.

Equation (9) shows that the modal overlap effect can be restated as a simple filtering formulation of a random signal characterized by a PSD $|\tilde{\gamma}(f)|^{2}$ applied to a filter with a frequency response $\left|\psi_{0}\left(f-f_{w}\right)\right|^{2}$.

The approach presented in this section can be extended to other statistical moments. For instance, it is easy to show that $\mathrm{E}\left[W^{2}\right]$ can be restated in a similar way to (9) where the filter response would be $\left|\psi_{0}\left(f-f_{w}\right)\right|^{4}$ and the PSD would be $|\tilde{\gamma}(f)|^{4}$ with an average value $\mu_{4}=\mathrm{E}\left[\left|\tilde{\gamma}_{n}\right|^{4}\right]$.

Note that if one is interested in the derivation of relative or normalized variance of $W$, one will have to deal with the following typical ratio,

$$
\mathcal{R}=\frac{<|\tilde{\gamma}(f)|^{4}\left|\psi_{0}\left(f-f_{w}\right)\right|^{4} \mid>_{\mathcal{F}}}{\left(<|\tilde{\gamma}(f)|^{2}\left|\psi_{0}\left(f-f_{w}\right)\right|^{2} \mid>_{\mathcal{F}}\right)^{2}},
$$


where $\left\langle\cdot>_{\mathcal{F}}\right.$ stands for the frequency average as defined in (9).
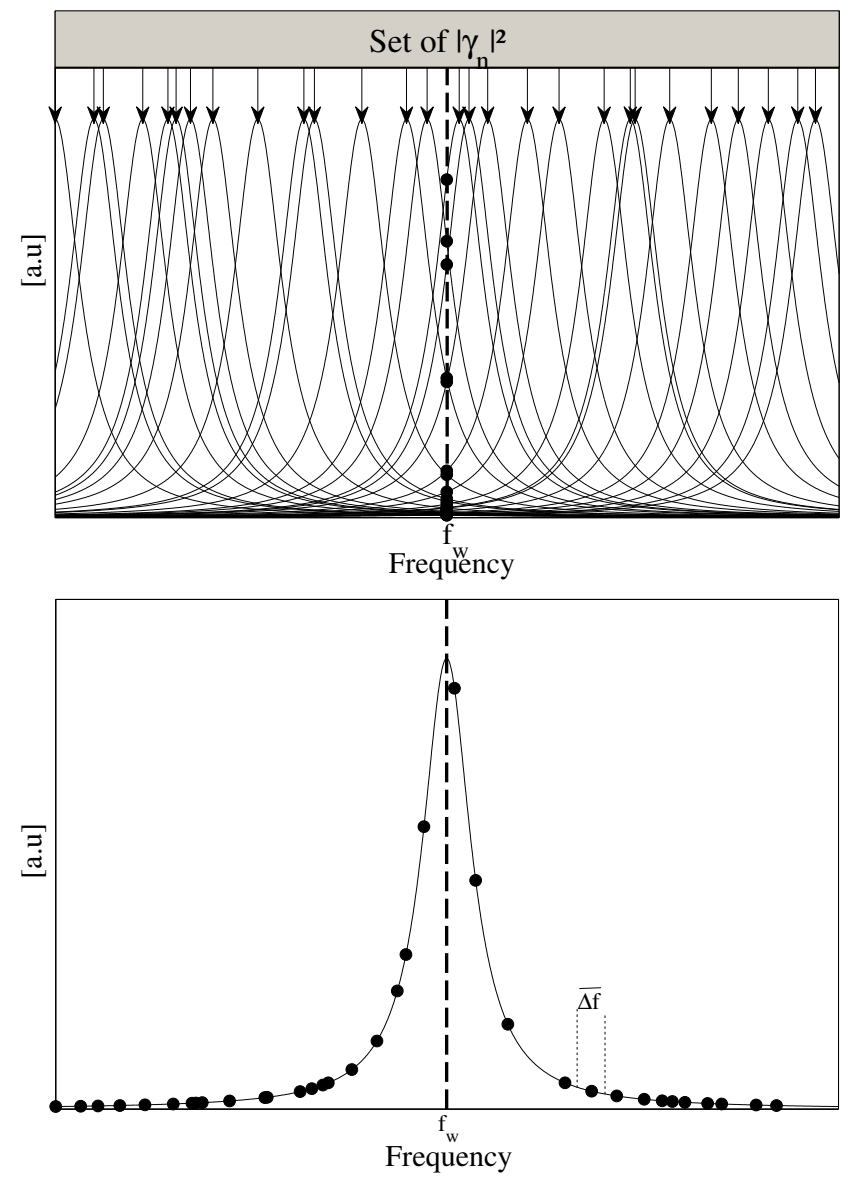

Fig. 1. Due to modal overlap all resonant frequencies $f_{n}$ are involved in the ensemble statistics of a given field-related quantity at given working frequency $f_{w}$ (black dots in the upper plot). Each frequency response is weighted by a corresponding $\left|\gamma_{n}\right|^{2}$ (vertical arrows). As illustrated, the black dots of the upper plot are contained in the template $\left|\psi_{0}\left(f-f_{w}\right)\right|^{2}$ (lower plot). This illustrates the ergodic principle stating that expected values on the eigenfrequencies ensemble converges to the one found using a single-mode frequency response in the whole frequency domain.

\section{STATISTICAL BANDWIDTH AND SIGNIFICANT MODES}

According to the previous section $\left|\psi_{0}\left(f-f_{w}\right)\right|^{2}$ and $\left|\psi_{0}\left(f-f_{w}\right)\right|^{4}$ can be regarded as weighting functions acting on $|\tilde{\gamma}(f)|^{2} \mid$ and $|\tilde{\gamma}(f)|^{4}$, respectively.

Weighting functions are common tools in signal processing and instrumentation. A classic application deals with the estimation of the PSD of a signal [13] based on analog signal processing. The estimation of the PSD at a given frequency $f_{w}$ consists in measuring the power of the signal at the output of a perfect filter, i.e., a filter with an ideally flat frequency response of width $B_{1}$. Due to the finite bandwidth of the filter, the estimation error is unavoidable and is characterized by its normalized relative error. In practice however, the filter used for such estimation is of Lorentzian shape and the resulting estimated error may vary. To relate the impact of using such filter instead of the ideal one, the concept of statistical bandwidth, referred to as $B_{s}$, is introduced. The latter corresponds to the bandwidth $B_{1}$ that would provide the same RV than the one obtained in practice.

Although the application is different in the present context, the scenario is quite similar if the normalized relative error is regarded as the relative variance (RV) of an unbiased estimator. Moreover, as stated previously, $|\tilde{\gamma}(f)|^{2}$ being the PSD of a random signal, we can easily show that the RV to compute corresponds to $\mathcal{R}$ given by (10). By using the statistical bandwidth concept it follows that,

$$
\mathcal{R}=\overline{\frac{\Delta f}{B_{s}}} \frac{\mu_{4}}{\mu_{2}^{2}},
$$

where $B_{s}$ is the statistical bandwidth defined as [13] [14],

$$
B_{s}=\frac{\left(\int_{0}^{\infty}\left|\psi_{0}\left(f-f_{w}\right)\right|^{2} \mathrm{~d} f\right)^{2}}{\int_{0}^{\infty}\left|\psi_{0}\left(f-f_{w}\right)\right|^{4} \mathrm{~d} f} .
$$

Considering the frequency response given by (5) we obtain,

$$
B_{s}=\pi B_{M} \text {. }
$$

The interest of the statistical bandwidth lies in the fact that the contribution, in a statistical sense, of an infinite number of $\left|\tilde{\gamma}_{n}\right|^{2}$ weighted by a Lorentzian spreading on a infinite frequency range, can be summed up by a finite number of un-weighted $\left|\tilde{\gamma}_{n}\right|^{2}$ in a finite bandwidth $B_{s}$.

Within the statistical bandwidth model, it follows that the number of significant modes, referred to as $M$, reads finally,

$$
M=\pi M_{M},
$$

where $M_{M}$ refers to the number of modes overlapping in the modal bandwidth $B_{M}$ that can be expressed as,

$$
M_{M}=m(f) B_{M},
$$

where $m(f)$ is the modal density in $\mathrm{Hz}^{-1}$.

The condition $V(f / c)^{3} \gg 1$ being met, the simplest form of the modal density [15] can be used, such that

$$
m(f) \simeq \frac{8 \pi V f^{2}}{c^{3}}
$$

where $V$ and $c$ are the volume of the MSRC and the speed of light, respectively.

It is worth stressing that the modal density is, in a strict way, a fluctuating quantity whose variance will not be taken into account in the present work. We will only consider its approximated median value given by (16).

\section{ASSESSMENT OF $M$}

The concept and the number of significant modes is difficult to validate since it cannot be "counted" in practice. However, as shown in [10] the variability of $W$ is governed by the number of modes $M_{M}$. With the concepts introduced in the previous sections, studying the variability of $W$ consists in assessing $\varsigma_{W}^{2}$ for a finite number $M$ of modes spreading over $B_{s}$. This is exactly the result given by Eq.(24) in [10], where $B_{e}$ was at that stage regarded as a finite bandwidth over which 
$M$ modes spread. Adapting the result is then straightforward, yielding,

$$
\begin{aligned}
\varsigma_{W}^{2} & =\frac{B_{s}}{M} \frac{\mu_{4}}{\mu_{2}^{2} \pi B_{M}}+\frac{M-4}{3 M} \\
& =\frac{1}{3}+\frac{2}{3 \pi M_{M}},
\end{aligned}
$$

for $\mu_{4} / \mu_{2}^{2}=2$, keeping the assumption that $\tilde{\gamma}_{i}$ are normallydistributed complex random variables.

A quick glance at the final expression of $\varsigma_{W}^{2}$ in [10] shows a difference of a factor 3 in the second term of the right-hand term. In fact, in order to include all the modes the initially finite bandwidth $B_{e}$ was logically extended to infinity, but $M / B_{e}$ was improperly substituted by the modal density $m(f)$ - indeed this substitution can only be justified if $B_{e}$ is sufficiently small. This is the advantage provided by the statistical-bandwidth concept which, given the order of magnitude of $B_{s}$, allows substituting $B_{s} / M$ by $1 / m(f)$ in (17).

Since $B_{s}$ and $M$ only exist in the framework of an equivalent statistical model, the validation of these as pertinent (and useful) quantities cannot be performed, but indirectly, by validating the analytical expression of $\varsigma_{W}^{2}$.

\section{CONCEPTS AND MODEL VALIDATION}

In order to validate the analytical expression of $\varsigma_{W}^{2}$ found in (17), we will compare the relative variance to the one obtained, on the one hand, by Monte Carlo (MC) simulations and on the other hand, experimentally.

\section{A. Monte Carlo simulation setup}

The importance of the results obtained by MC simulation should not be underestimated over those obtained experimentally. Indeed, beyond the flexibility that the MC method allows, it is a very convenient way to check the self-consistency of our analytical expressions with respect to the assumptions made on the different parameters intervening in (3).

The statistical distributions are such that the real and imaginary parts of the equivalent modal weights were assumed to follow a Normal law; a uniform distribution was assumed for both the polarization of the modes, over $4 \pi$ sr.

As stated by (17), the only parameter that can vary is the number of modes overlapping in the modal bandwidth $B_{M}$. As shown by (16), this number can be modified by considering a variable volume and/or a variable frequency. As shown in [16], the two approaches are equivalent. In order to simplify MC computations, we pick out the method consisting in fixing a modal bandwidth and adapting a (virtual) volume to ensure the desired number of overlapping modes. In the present work a $1-\mathrm{MHz}$ modal bandwidth is arbitrarily set.

Each MC simulation consisted in generating twenty sets of 50000 independent random realizations of the electric field described by (3), and this for the following values of $M_{M}$ : 1, $2,3,5,10,15,20,25,30,35$. The number of simulated modes was taken over a bandwidth of $51 B_{M}$ [16]. The resulting estimated variance was averaged over the twenty values.

\section{B. Experimental setup and chamber characterization}

In order to make the comparison more sensible with [10], the same measurements are considered herein. As a brief reminder, and to provide some more details, the setup takes place in the $13.3 \mathrm{~m}^{3}(3.08 \mathrm{~m} \times 1.84 \mathrm{~m} \times 2.44 \mathrm{~m}) \mathrm{RC}$ equipped with a 100-step mechanical stirrer blade of $50 \mathrm{~cm}$ wide; its LUF is around $550 \mathrm{MHz}$. The relative variance is studied over the frequency range of $0.7-3 \mathrm{GHz}$.

The RC was used in two configurations. In the first one the $\mathrm{RC}$ was empty; in the second one it was loaded by inserting an hybrid absorber made up of four pyramids of about $30 \mathrm{~cm}$ high, standing in the center of the RC.

The loaded and empty cases provide respective advantages. When the RC is not loaded, losses are minimized, allowing us to visualize, as clearly as possible, the transition of $\varsigma_{W}^{2}$ towards its well-known asymptotic value of $1 / 3$. The interest of the loaded case is to provide another configuration of the chamber and to have enough overlapping modes in order to approach the asymptotic value, i.e., to attain very wellovermoded conditions.

For the empty and loaded scenarios, we can show that the maximum average modal bandwidths are $150 \mathrm{kHz}$ and $375 \mathrm{kHz}$, respectively. On the frequency range of interest, 1000 linearly-spaced frequency bins are used, inducing a $2.3 \mathrm{MHz}$ frequency space between each point. This frequency space is much larger than the maximum value of the modal bandwidths previously mentioned, allowing each measured point to be considered as uncorrelated between one another.

The variability $\varsigma_{W}^{2}$ will be estimated with an inevitable uncertainty. The latter, i.e., the uncertainty, can be minimized by applying a moving average over 5 contiguous points, followed by a decimation whose factor equals 5 accordingly. Average values are therefore obtained over $10-\mathrm{MHz}$ bandwidths.

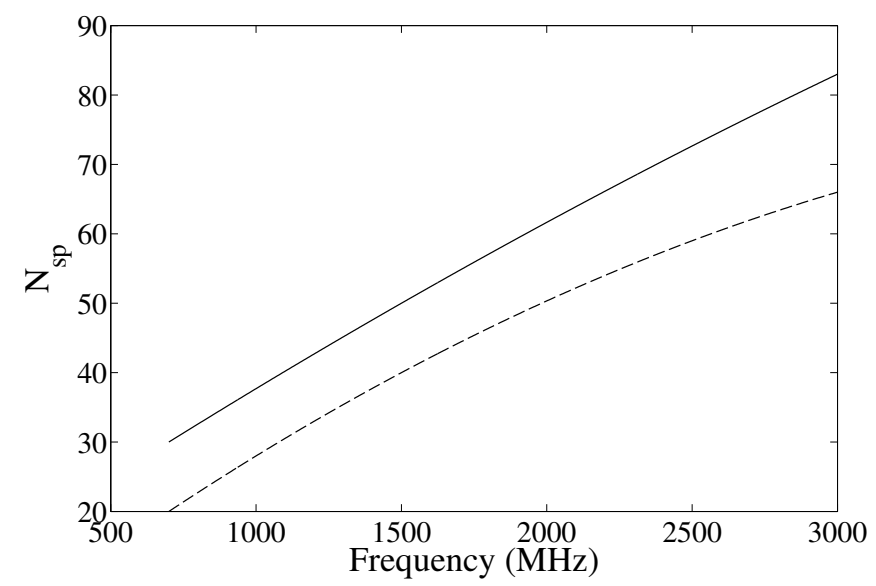

Fig. 2. Estimated number of independent stirrer positions as a function of frequency for the empty (solid line) and the loaded (dashed line) cases, respectively.

It is worth recalling that modal bandwidths and the modal density are two parameters that can fluctuate considerably. The moving average followed by the decimation aim at extracting the average trend, in order to be consistent with the model used herein that neither takes into account the variance of the 
number of modes [17] nor the variance of the quality factors [18] [15].

In order to make the validation reliable, we need to estimate some useful preliminary quantities such as the number of uncorrelated stirrer positions, referred to as $N_{s p}$.

The number of uncorrelated stirrer positions can be assessed when the correlation coefficient is below a certain threshold that depends on the number of stirrer positions [1]. For 100 positions the threshold is found around $20 \%$. For this correlation level, Fig. 2 shows the resulting number of "independent" number of stirrer positions as a function of frequency for the empty (solid line) and loaded (dashed line) scenarios, respectively.

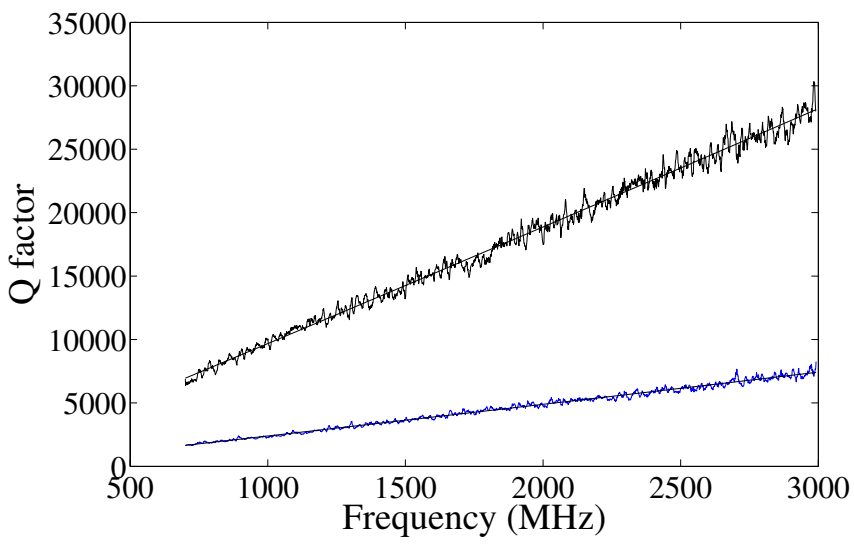

Fig. 3. Composite quality factor obtained by averaging over $10-\mathrm{MHz}$ bandwidths in the empty case (black upper curve) and the loaded case (grey lower curve), respectively. Straight lines stands for first-order approximated median values in a least-square sense.

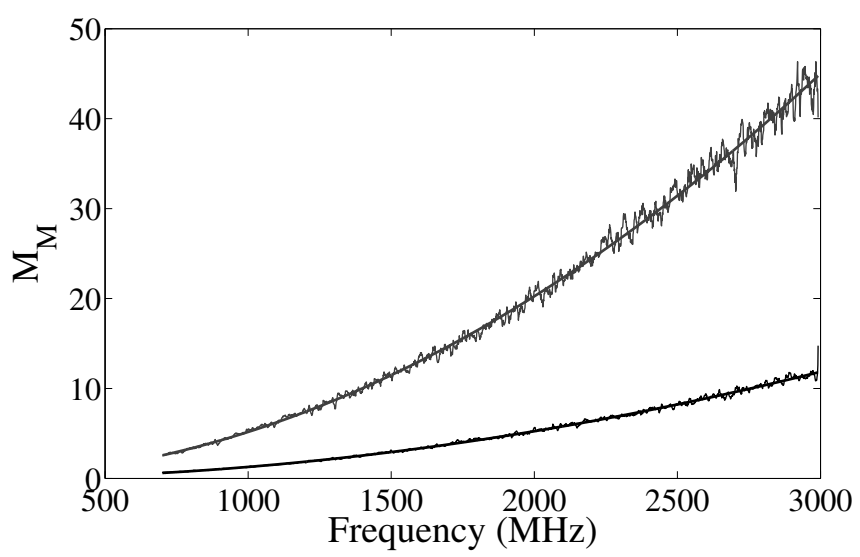

Fig. 4. Number of overlapping modes in the $-3-\mathrm{dB}$ bandwidth obtained when the RC is empty (black lower curve) and loaded (grey upper curve). Solid lines are first-order approximated median values in a least-square sense.

In the validation process, the number of overlapping modes $M_{M}$ is also a key quantity resulting from the knowledge of the composite quality factor. Accordingly, a special care must be taken with the estimation of $Q$. As the field probe used was phase sensitive, we were able to compute the composite quality factor for the chamber over the entire frequency range of test, by postprocessing the frequency-spectrum data in time domain. The frequency spectrum used was made up of 60000

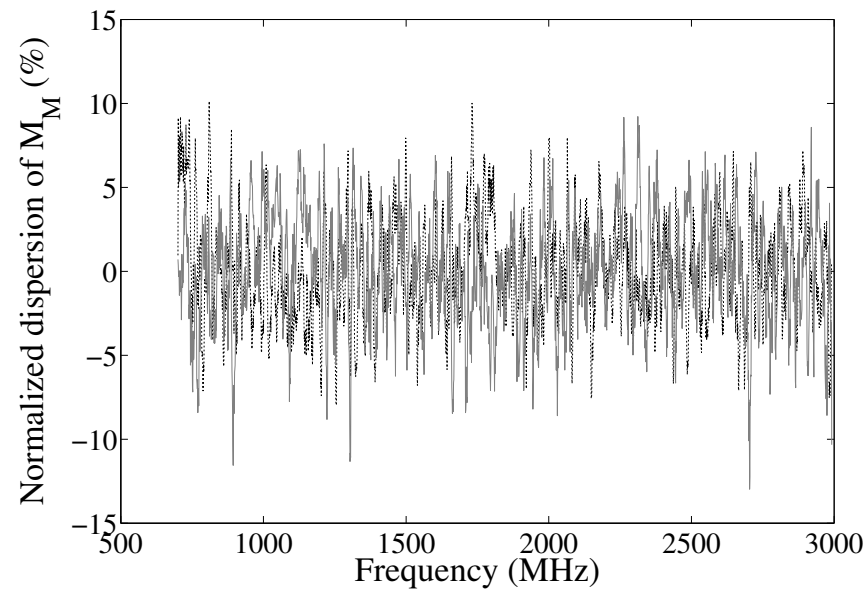

Fig. 5. Fluctuations of the estimated $M_{M}$ values, obtained in Fig. 4, about the approximated median values, for the empty case (solid grey line) and the loaded case (black dotted line), respectively.

bins over the entire frequency range of interest. Composite quality factors were estimated on $1-\mathrm{MHz}$ frequency bands. In order to be consistent with the process applied to $\varsigma_{W}^{2}, Q$ values were averaged on $10-\mathrm{MHz}$ frequency bands; the uncertainty was further minimized by using the data obtained on the three field components.

Fig. 3 shows the resulting composite quality factors obtained for the empty (black upper curve) and loaded cases (grey lower curve), respectively. In order to extract the mean trend for both cases, a first-order fit was performed in a least-square sense (straight lines). The measured quality factors, as a function of frequency, allow to compute modal bandwidths.

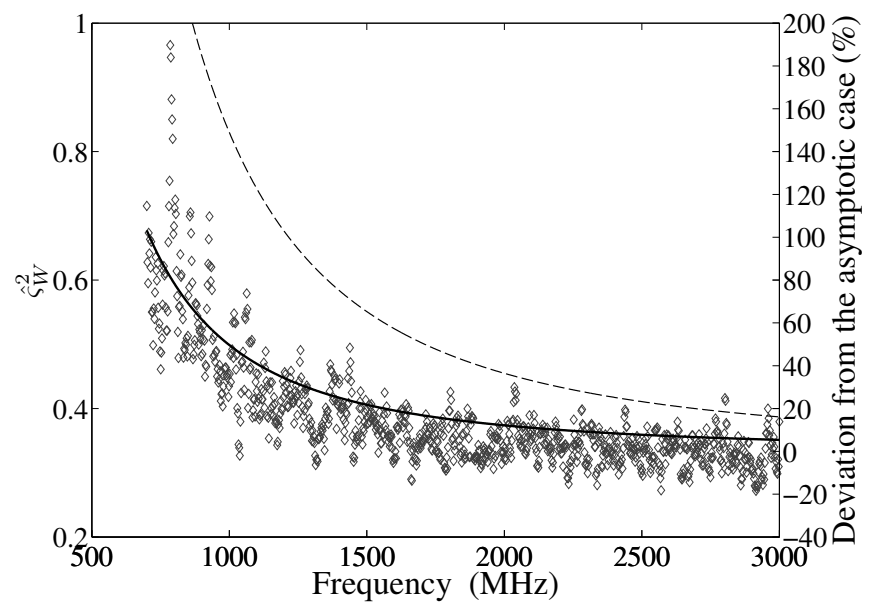

Fig. 6. Estimated relative variance $\hat{\varsigma}_{W}^{2}$ of the electric-energy density (left $y$-axis) and the relative deviation from the asymptotic value of $1 / 3$ (right $y$-axis), as a function of frequency. Experimental results (grey markers) and analytical results (solid line) are reported; dashed line stands for the analytical expression derived in [10].

The knowledge of (average) modal bandwidths allowed us to derive the number $M_{M}$ of overlapping modes by using (15). Accordingly, Fig. 4 shows the number $M_{M}$ for the empty (black lower curve) and loaded cases (grey upper curve), respectively. Solid lines provide the mean trends resulting from those obtained for $Q$. Note that the fluctuations of $M_{M}$ 
observed in Fig. 4 results from the fluctuations of the estimated composite quality factor and are not due to local modal-density fluctuations; as explained in section III, these have not been considered in the present work.

In order to have an estimation of the fluctuations of $M_{M}$ about mean values, Fig. 5 shows the normalized dispersion obtained for the empty case (solid grey line) and the loaded case (black dotted line), respectively. A dispersion of about $\pm 7 \%$ is observed for both cases.

This characterization of the chamber in both scenarios allows us to proceed to the validation of the relative variance of the electric-energy density.
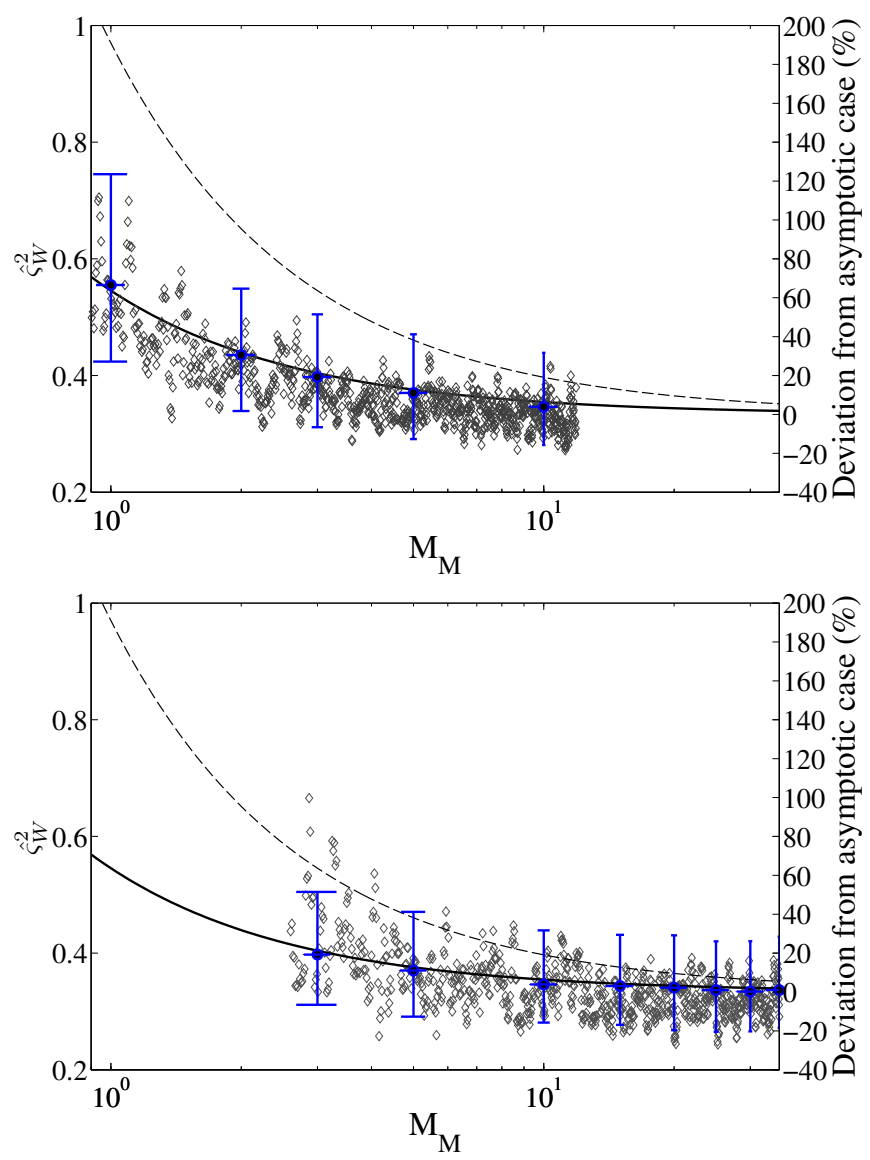

Fig. 7. Estimated relative variance $\hat{\varsigma}_{W}^{2}$ (left y-axis) of the electric-energy density, as a function of the number of modes $M_{M}$ for the empty (upper plot) and loaded (lower plot) scenarios, respectively. Corresponding relative deviation from the asymptotic value of $1 / 3$ (right y-axis). Experimental results (grey symbols), MC results (black dots) and analytical results (solid line) are reported. The dashed line stands for the analytical expression derived in [10] Vertical bars is related to the uncertainty of the experimental measurements considered on a 95\% confidence interval. Horizontal bars stands for the dispersion of $M_{M}$ around its mean value.

\section{Results}

We present in Fig. 6 the estimated values of $\varsigma_{W}^{2}$, referred to as $\hat{\varsigma}_{W}^{2}$, obtained experimentally (left y-axis) as a function of frequency (grey markers) with the corresponding relative deviation from its asymptotic value (right y-axis). For clarity, and in order to well visualize the transition of the relative variances towards their asymptotic values, only the empty case is reported in Fig. 6. We superimposed the analytical expression obtained in (17) (solid line) and the analytical result obtained in [10] (dashed line). We can observe that the expression given by (17) is in very good agreement with measurements, whereas the relative variance $\hat{\varsigma}_{W}^{2}$ obtained in [10] (dashed line) tends to overestimate the degree of nonuniformity of $W$.

In order to compare these results to those obtained with the MC approach, the number of modes assessed experimentally for the empty and loaded scenarios (see Fig. 4) must be used. This allows to transpose and superimpose the experimental results to those obtained by MC simulation. Variabilities are shown (left y-axis) accordingly in Fig. 7, where experimental results (grey markers), MC results (black dots) and analytical results (solid line) have been reported. Deviation of $\varsigma_{W}^{2}$ from its asymptotic value is also shown (right y-axis).

However, when experimental variances are estimated from sets made up of finite number of samples, directly related herein to the number $N_{s p}$ of independent stirrer positions, an unavoidable uncertainty has to be taken into account. To estimate the latter, MC simulations were used again; this merely consisted in computing 5000 times, for a given $M_{M}$ value, estimated relative variances of $W$ obtained over sets composed of $N_{s p}$ realizations.

Recalling that $\hat{\varsigma}_{W}^{2}$ plotted in Fig. 6 was deduced from averages performed over 5 contiguous points, the $5000 \mathrm{MC}$ values were rearranged in $5 \times 1000$ values in order to perform averages over 5 contiguous points. From these averages, $95 \%$ confidence intervals of $\hat{\varsigma}_{W}^{2}$ have been computed, and correspond to the uncertainty bars reported in Fig. 7, for the empty case (upper plot) and the loaded case (lower plot), respectively.

Finally, we need to recall that $M_{M}$ are mean estimated values for which $7 \%$ fluctuations were observed (see Fig. 5); accordingly, horizontal uncertainty bars on $M_{M}$ have been added in Fig. 7.

We can observe a very good agreement between the results obtained analytically, numerically and those obtained experimentally. For the latter, we note remarkable agreement even with uncertainty bars, especially for the empty case.

We stress the interest of using an MC approach, since the good agreement found ensures in some way that analytical results are consistent with the assumptions made on the different parameters and their related statistical laws.

\section{CONCLUSION}

The concept of significant modes has been revisited and highlighted. The bandwidth over which this number is defined, depends inevitably on the statistical quantity of interest. In the present work, a quantitative assessment of this number has been carried out on the basis of the variability of the electricenergy density. This notwithstanding, the approach presented in the present work can be extended to other statistical quantities.

Linking the concept of significant modes to previous work allowed us to derive a new expression of the variability of the electric-energy density. The good agreement found with experimental results and simulation supports the different concepts 
introduced in the present paper. From a more practical point of view, it provides an answer to the pending question dealing with the assessment of the average number of significant modes to consider at a given frequency in an MSRC.

\section{REFERENCES}

[1] Reverberation chamber test methods, International Electrotechnical Commission (IEC), Std. 61 000-4-21, 2011.

[2] J. Kostas and B. Boverie, "Statistical model for a mode-stirred chamber," IEEE Transactions on Electromagnetic Compatibility, vol. 33, no. 4, pp. 366-370, 1991.

[3] M. Hoijer, "Maximum power available to stress onto the critical component in the equipment under test when performing a radiated susceptibility test in the reverberation chamber," IEEE Transactions on Electromagnetic Compatibility, vol. 48, no. 2, pp. 372-384, 2006.

[4] C. Lemoine, P. Besnier, and M. Drissi, "Investigation of reverberation chamber measurements through high-power goodness-of-fit tests," IEEE Transactions on Electromagnetic Compatibility, vol. 49, no. 4, pp. 745 755, 2007.

[5] —_ "Estimating the effective sample size to select independent measurements in a reverberation chamber," IEEE Transactions on Electromagnetic Compatibility, vol. 50, no. 2, pp. 227-236, 2008.

[6] R. Serra, F. Leferink, and F. Canavero, “"'Good-but-imperfect” electromagnetic reverberation in a VIRC," in Electromagnetic Compatibility (EMC), 2011 IEEE International Symposium on, 2011, pp. 954-959.

[7] C. F. Bunting, "Statistical characterization and the simulation of a reverberation chamber using finite-element techniques," IEEE Transactions on Electromagnetic Compatibility, vol. 44, no. 1, pp. 214-221, 2002.

[8] L. Arnaut, "Compound exponential distributions for undermoded reverberation chambers," IEEE Transactions on Electromagnetic Compatibility, vol. 44, no. 3, pp. 442-457, 2002.

[9] G. Orjubin, E. Richalot, S. Mengue, and O. Picon, "Statistical model of an undermoded reverberation chamber," IEEE Transactions on Electromagnetic Compatibility, vol. 48, no. 1, pp. 248-251, 2006.

[10] A. Cozza, "The role of losses in the definition of the overmoded condition for reverberation chambers and their statistics," IEEE Transactions on Electromagnetic Compatibility, vol. 53, no. 2, pp. 296-307, 2011.

[11] L. Arnaut, "Limit distributions for imperfect electromagnetic reverberation," IEEE Transactions on Electromagnetic Compatibility, vol. 45, no. 2, pp. 357-377, 2003.

[12] B. Liu, D. Chang, M. Ma, and U. S. N. B. of Standards, Eigenmodes and the composite quality factor of a reverberating chamber. National Bureau of Standards, 1983.

[13] J. Bendat and A. Piersol, Random data: analysis and measurement procedures. Wiley-Interscience, 1971.

[14] W. Stanley and S. Peterson, "Equivalent statistical bandwidths of conventional low-pass filters," Communications, IEEE Transactions on, vol. 27, no. 10, pp. 1633-1634, 1979.

[15] L. Arnaut and G. Gradoni, "Probability distribution of the quality factor of a mode-stirred reverberation chamber," IEEE Transactions on Electromagnetic Compatibility, vol. 55, pp. 35-44, 2013.

[16] F. Monsef, "Why a reverberation chamber works at low modal overlap," IEEE Transactions on Electromagnetic Compatibility, vol. 54, no. 6, pp. 1314-1317, 2012.

[17] A. Cozza, "Probability distributions of local modal-density fluctuations in an electromagnetic cavity," IEEE Transactions on Electromagnetic Compatibility, vol. 54, no. 5, pp. 954 - 967, 2012.

[18] L. Arnaut, "Statistics of the quality factor of a rectangular reverberation chamber," IEEE Transactions on Electromagnetic Compatibility, vol. 45, no. 1, pp. 61-76, 2003.

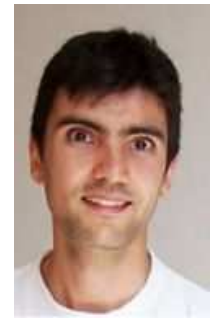

Florian Monsef received his Master Degree from the Université Paris-Sud, Orsay, France. He entered the Ecole Normale Supérieure de Cachan in electrical engineering and computer science. He did a thesis on electron transport in IV-IV heterostructures and obtained his $\mathrm{PhD}$ in electronics from Université Paris-Sud, Orsay, France, in 2002. From 2002 to 2009 he was a teacher in electrical engineering at the Technical Institute of the Université Paris X. Since 2009 he is an assistant professor at the Laboratoire des Signaux et Systèmes (Université Paris-Sud). His topics in research since 2009 have been dealing with EMC, reverberation chambers and Time-Reversal applications.

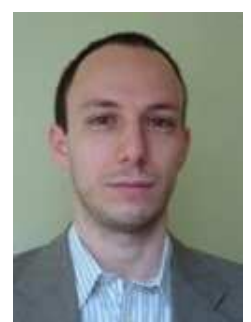

Andrea Cozza(S02M05) received the Laurea degree (summa cum laude) in electronic engineering from Politecnico di Torino, Turin, Italy, in 2001, and the $\mathrm{Ph} . \mathrm{D}$. degree in electronic engineering jointly from Politecnico di Torino and the University of Lille, France, in 2005. In 2007, he joined the Département de Recherche en lectromagnétisme, SUPELEC, Gif sur Yvette, France, where since 2013 he is full professor. He is a reviewer for several scientific journals, including those of IET and IEEE. His current research interests include reverberation chambers, statistical electromagnetics, wave propagation through complex media and applications of time reversal to electromagnetics. Dr. Cozza was awarded the 2012 Prix Coron-Thévenet by the Académie des Sciences, in France. 
Description of the changes in the final manuscript

An sentence has been added in the $2 \S$ of the introduction

"An important part of the measured-field uncertainty is directly due to the medium complexity"

The two last sentences of the introduction has been reformulated as follows

"However, the number of significant modes cannot be directly assessed experimentally. This notwithstanding, as shown insection IV, the validation of $\zeta_{w}{ }^{\prime}$ s law allows us to validate the assessment of the number of significant modes."

For clarity equation (1) has been recast in a more conventional way. The text following (1) has been restated as follows,

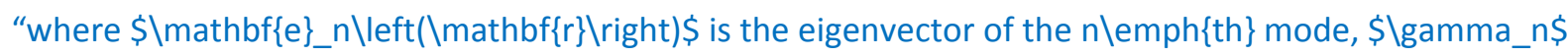
is the modal weight, i.e., the coupling constant of the excitation source to the $\$ n \$$ th eigenmode and \$psi_n $n$ is the frequency response of the $\$ n \$$ th mode.

If we express, on the one hand,

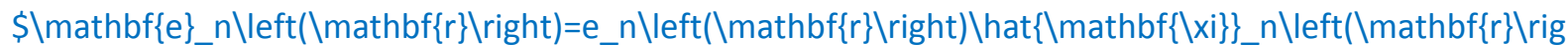
$h t) \$$ where $\$ \backslash$ hat $\{\backslash$ mathbf $\{\backslash x i\}\} \_n \backslash$ left $(\backslash$ mathbf $\{r\} \backslash$ right $) \$$ is the unitary polarization vector of the

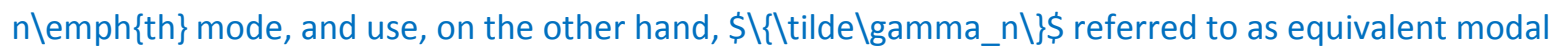
weights and defined as,"

The ex Equation (1) has been displaced. Its label is now equation (3).

In the first column of page 2 last paragraph "black-dots values" replaced by "values indicated by the black dots"

For the sake of improving the grammar and style the paragraph following equation (7) has been rewritten as follows

"In order to further recast (6), it is worth recalling that the ensemble average operator stands for an average over an ideally infinite number of stirring states. Moreover, since a stirring process consists in displacing resonance frequencies over a small frequency range, referred to as $\$$ loverline $\{\backslash$ Delta $f\} \$$ (see lower plot of Fig. 1, the vertical dashed line in the upper plot of Fig. 1, as well as the initially discrete frequency template, will both become continuous under perfect stirring conditions.

Note that the random signal $\$ \mid \backslash$ tilde $\mid$ gamma $\backslash$ left(f) $\backslash$ right $)\left.\right|^{\wedge} 2 \$$ is analog to a power spectrum density (PSD). This one will become continuous in the frequency domain with an average amplitude

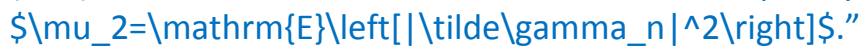

After equation (8) (now eq.(9)) the sentence was recast as follows

"where $B_{e}$ is the frequency range centered about $f_{w}$ an infinite $B_{e}$ allows including all the modes."

The acronym PSD has been been added in section II to make the link clearer with concepts introduced in section III. Accordingly, $|\gamma(f)|^{2}$ is referred to as a PSD in the paragraph preceding eq.(9). 
The text "Relation (8) exhibits another point showing that the modal overlap effect in the computation of the $E[W]$ can be restated as a simple filtering problem of a random signal $|\sim(f)| 2 \mid$; the frequency response of the filter being $\left|\psi_{0}(f-f w)\right|^{2}$." Has been replaced by

"Equation (9) shows that the modal overlap effect can be restated as a simple filtering formulation of a random signal characterized by a PSD $|\gamma(f)|^{2}$ applied to a filter with a frequency response $\left|\psi_{0}\left(f-f_{w}\right)\right|^{2}$."

The text before $\mathrm{Eq}(9)$ (now $\mathrm{Eq}(10)$ ) has been restated as follows

"Note that if one is interested in the derivation of relative or normalized variance of W, one will have to deal with the following typical ratio,"

In section III the first paragraph has been split into 2 paragraphs for clarity. The following statement has been deleted "error, which, for an unbiased estimator, can be regarded as a relative variance (RV)."

The paragraph preceding eq (10) (now eq (11)) has been restated as follows

"Although the application is different in the present context, the scenario is quite similar if the normalized relative error is regarded as the relative variance (RV) of an unbiased estimator. Moreover, as stated previously, $|\gamma(f)| 2$ being the PSD of a random signal, we can easily show that the RV to compute corresponds to R given by (10). By using the statistical bandwidth concept it follows that,"

Paragraph preceding eq.(15) (now eq.(16)) has been shortened as follows,

"The condition V $(\mathrm{f} / \mathrm{c})^{3} \gg>1$ being met, the simplest form of the modal density [15] can be used, such that"

For the sake of clarity the following sentence has been added at the end of section III

"Since Bs and $\mathrm{M}$ only exist in the framework of an equivalent statistical model, the validation of these as pertinent (and useful) quantities cannot be performed, but indirectly, by validating the analytical expression of $\varsigma^{2} w . "$

The two first paragraphs of the conclusion has been joined by shortening the first (ex-) first paragraph. It follows that the first paragraph is such that,

"The concept of significant modes has been revisited and highlighted. The bandwidth over which this number is defined, depends inevitably on the statistical quantity of interest. In the present work, a quantitative assessment of this number has been carried out on the basis of the variability of the electric-energy density. This notwithstanding, the approach presented in the present work can be extended to other statistical quantities." 